\title{
Call for a standard unit of defect size
}

\author{
Frans J. Th. Wackers, MD \\ Section of Cardiovascular Medicine, Yale University, New Haven, CT \\ Received Feb 12, 2014; accepted Feb 13, 2014 \\ doi: $10.1007 /$ s12350-014-9874-8
}

\section{See related article, pp. 673-681}

Until the late eighteenth century the units of length varied in different countries, even from city to city. Units of length were traditionally based on the dimensions of the human body: foot, underarm or thumb. An American foot was $305 \mathrm{~mm}$, the French foot $324 \mathrm{~mm}$ and the Dutch foot $283 \mathrm{~mm}$. $^{1}$ Attempts to standardize these measures were largely unsuccessful. A similar lack of standardization existed for units of weight, volume, and time. This lack of uniformity presented serious problems for national and international trade.

For too long, interpretation of radionuclide stressrest myocardial perfusion imaging has been based largely on visual analysis alone. Realizing that it was not adequate to interpret radionuclide images as just normal or abnormal, semi-quantitative interpretation was introduced in the 1980s. Images of the left ventricle were divided into segments and each segment was scored according to the visually perceived degree of decreased radiotracer uptake. The derived summed score expressed the extent of abnormality. In older publications, one can find any number of segments as well scoring methods. In 2002 and 2006 ASNC guidelines recommended the current standard 17-segment and 0-4-point scoring system. ${ }^{2,3}$ Although this represents an improvement, the methodology is still subjective.

Only recently expert analyses of paired stress myocardial perfusion images in multicenter studies have shown unequivocally that visual analysis, even by very experienced readers, is seriously flawed by suboptimal inter- and intra-observer reproducibility. ${ }^{4,5}$

For more than two decades quantitative computer software for radionuclide myocardial perfusion imaging

Reprint requests: Frans J. Th. Wackers, MD, Section of Cardiovascular Medicine, Yale University, New Haven, CT; frans.wackers@yale.edu. J Nucl Cardiol 2014;21:682-4.

1071-3581/\$34.00

Copyright (C) 2014 American Society of Nuclear Cardiology. has been commercially available. Using this software the computer performs segmentation and quantifies abnormal radiotracer uptake. These software packages have shown excellent reproducibility. Although in many laboratories the software is used for image processing and display, in practice the numbers generated by quantification is rarely used in reports.

In 1997, we presented preliminary data ${ }^{6}$ of a comparison of quantification of SPECT defect sizes by four different software programs, as well classification of defect abnormalities by four well-known laboratories and experts: Cedars-Sinai Medical Center (Berman), Allegheny Center for Health Science (Iskandrian), Baylor College of Medicine (Verani), and Yale University (Wackers).

Raw data (without attenuation correction) of 50 stress-rest SPECT studies were sent to the centers to be processed using their own clinical software. In addition, the experts were asked to classify stress defects as none, small, moderate, or large. Although there was a reasonable overall correlation of quantitative and visual defect sizes (coefficients ranging from $r=0.88$ to $r=0.91$ ), there was substantial spread of individual data points (Figure 1A-C) particularly for large defects. The different software programs showed systematic over- or underestimation of defect sizes when compared to each other. When the expert readers categorized the stress defects, there was poor agreement (Figure 1D), particularly for small defects. Thus, although there was a good relative correlation of quantified defect sizes, no such agreement was achieved for absolute measurements and classification.

We did not publish these results because we felt that they were embarrassing for the field of nuclear cardiology. We recommended that a laboratory should use only one type of quantitative software to assure consistency and reproducibility of interpretation.

Now, 15 years later, Ather et al. ${ }^{7}$ in the current issue of the Journal report similar results of a similar comparative analysis: significant differences between software programs in measuring perfusion defect sizes, as well as LV function. They also noted that 


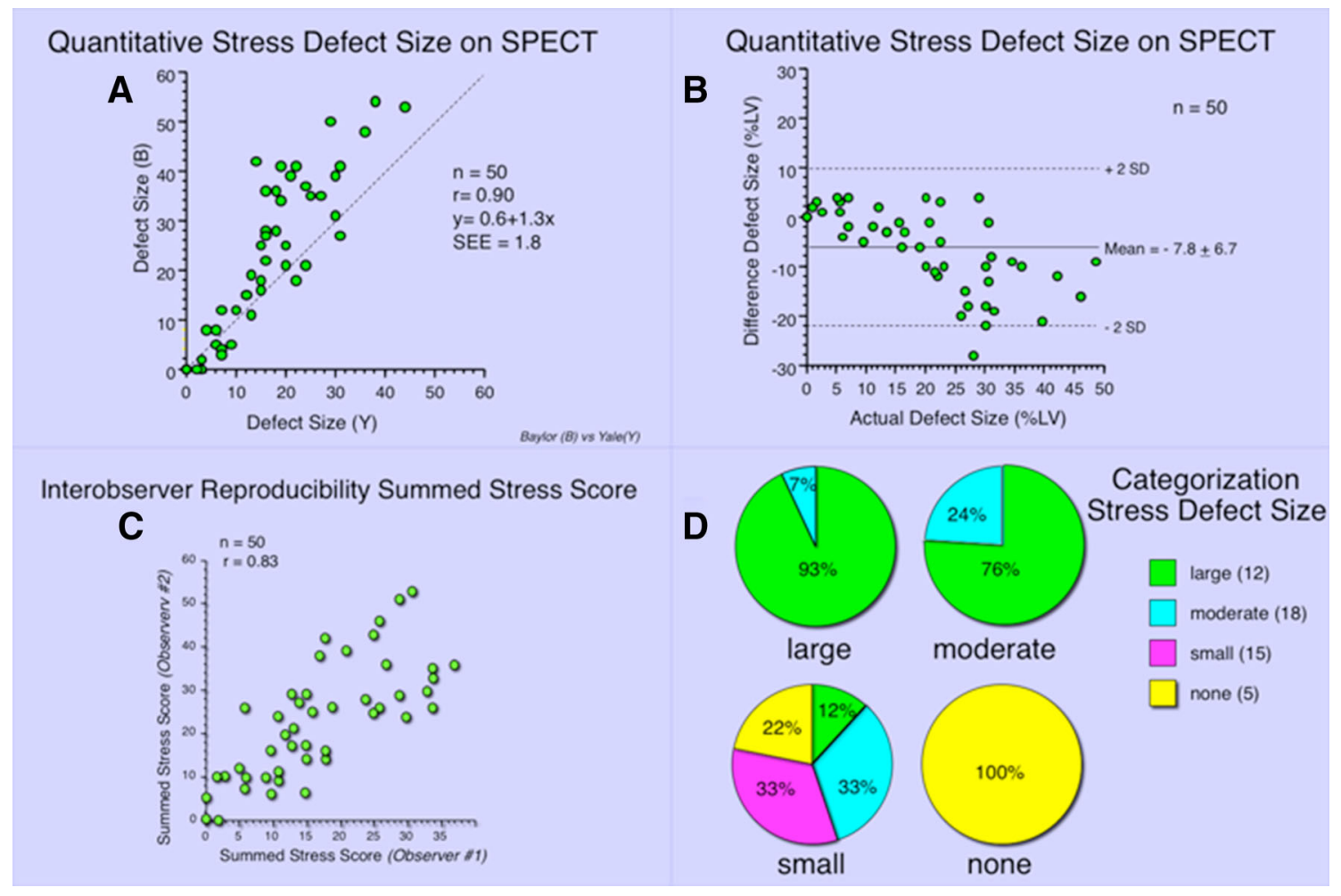

Fig. 1. Data from the 1997 comparison of four quantitative software programs. (A, B) representative correlation and Bland-Altman analysis of agreement between the Baylor and Yale programs. Although overall correlation coefficient was good, there was a systematic overestimation of defect sizes by the Baylor program. (C) Comparison of summed stress scores between two observers. Although overall correlation coefficient was good, there were substantial disagreements for individual scores. (D) Representative example of comparison of categorization of stress defect sizes by two readers. There is good agreement for studies with no and large defects. However, agreement was poor and fair respectively for small defects and moderate defects.

classification of defects as small, moderate or defect sizes had particularly poor concordance.

I believe that the time has come for the field of nuclear cardiology to act and to correct an embarrassing state of affairs. Nuclear cardiology should adopt a uniformly accepted independent standard for myocardial perfusion defect size.

After the French revolution, a committee chose arbitrarily the meter as the basic unit of length. It was determined that the meter was to be equal to "the ten millionth part of one quarter of the terrestrial meridian." A platinum meter standard bar still rests in the Archives of the French Republic in Paris. More practically, in 1799 a standard bar carved in marble, representing the meter (Figure 2), was mounted in the walls of 16 Parisian markets and public places as a reference "for all times and all men."

A similar initiative should now be taken regarding SPECT myocardial perfusion defect sizes.

This could be done in two steps. First, by creating a standard cardiac phantom with fillable defect inserts of

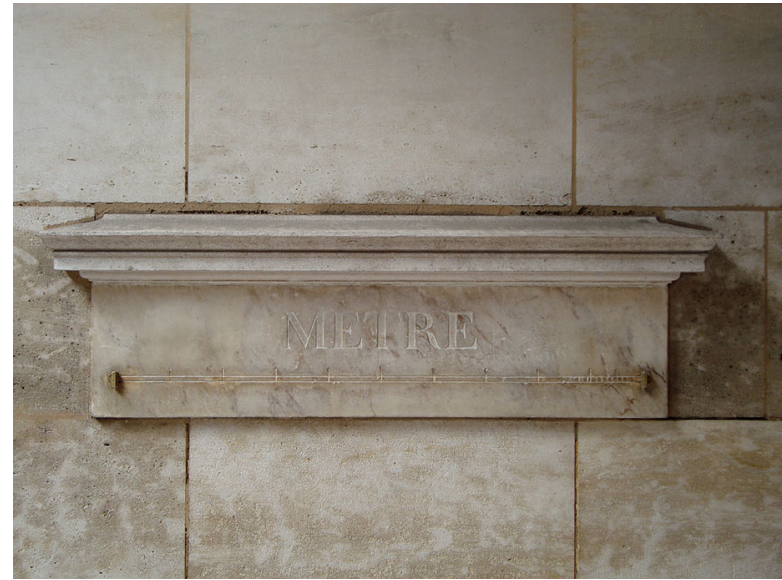

Fig. 2. Standard meter carved in marble in the wall of a public arcade at 36 Rue de Vaugirard, Paris, France.

known volumes to test accuracy of measurements by the various programs. ${ }^{8}$ Next, by creating a "depot" of benchmark SPECT myocardial perfusion studies with 
perfusion abnormalities that experts by consensus have designated to be small, moderate, and large. Vendors should then be required to adjust their software measurements in such a way that their quantitative data are in agreement with the benchmark studies.

I call upon the Quality Committee of the American Society of Nuclear Cardiology to establish a Standard Unit of Defect Size for myocardial perfusion imaging.

Since the magnitude of ischemic burden on stress myocardial perfusion imaging has an important discriminate and prognostic value, a uniformly accepted unit of defect size is crucial for responsible patient care.

\section{References}

1. Foot (unit)-Wikipedia, the free encyclopedia. http://en.wikipedia. org/wiki/Foot_\%28unit\%29 Accessed Feb 10, 2014.

2. Cerqueira MD, Weissman NJ, Dilsizian V, et al. Standardized myocardial segmentation and nomenclature for tomographic imaging of the heart: A statement for healthcare professionals from the cardiac imaging committee of the council on clinical cardiology of the American Heart Association. J Nucl Cardiol 2002;9:539-42.
3. Hansen CL, Goldstein RA, Berman DS, et al. Myocardial perfusion and function single photon emission computed tomography. J Nucl Cardiol 2006;13:e97-120.

4. Iskandrian AE, Bateman TM, Belardinelli L, Blackburn B, Cerqueira MD, Hendel RC, et al. Adenosine versus regadenoson comparative evaluation in myocardial perfusion imaging: Results of the ADVANCE phase 3 multicenter international trial. J Nucl Cardiol 2007;14:645-58.

5. Udelson JE, Heller GV, Wackers FJTh, Chai A, Hinchman D, Coleman PS, et al. Randomized, controlled dose-ranging study of the selective adenosine A2A receptor agonist Binodenoson for pharmacological stress a an adjunct to myocardial perfusion imaging. Circulation 2004;109:457-64.

6. Wackers FJT, Iskandrian AE, Verani MS, Berman DS, Natale D, Sinusas AJ, VanTrain KF, He ZX, DeMan P. Multicenter comparison of quantification of SPECT defect size using different quantitative approaches (Abstract). Circ Suppl 1997;96:309I.

7. Ather S, Iqbal F, Gulotta J, Aljaroudi W, Heo J, Iskandrian AE, Hage FG. Comparison of three commercially available softwares for measuring left ventricular perfusion and function by gated SPECT myocardial perfusion imaging. J Nucl Cardiol 2014. doi: 10.1007/s12350-014-9885-5.

8. Liu YH, Sinusas AJ, DeMan P, Zaret BL, Wackers FJTh. Quantification of SPECT myocardial perfusion images: methodology and validation of the Yale-CQ method. J Nucl Cardiol 1999;6:190-204. 